\title{
THE VELOCITY DISTRIBUTION OF SOLAR PHOTOSPHERIC MAGNETIC BRIGHT POINTS
}

\author{
P. H. Keys ${ }^{1}$, M. Mathioudakis ${ }^{1}$, D. B. Jess ${ }^{1}$, S. Shelyag ${ }^{1}$, P. J. Crockete ${ }^{1}$, D. J. Christian ${ }^{2}$, and F. P. Keenan ${ }^{1}$ \\ ${ }^{1}$ Astrophysics Research Centre, School of Mathematics and Physics, Queen's University, Belfast BT7 1NN, UK \\ ${ }^{2}$ Department of Physics and Astronomy, California State University, Northridge, CA 91330, USA \\ Received 2011 August 4; accepted 2011 September 7; published 2011 September 28
}

\begin{abstract}
We use high spatial resolution observations and numerical simulations to study the velocity distribution of solar photospheric magnetic bright points. The observations were obtained with the Rapid Oscillations in the Solar Atmosphere instrument at the Dunn Solar Telescope, while the numerical simulations were undertaken with the MURaM code for average magnetic fields of $200 \mathrm{G}$ and $400 \mathrm{G}$. We implemented an automated bright point detection and tracking algorithm on the data set and studied the subsequent velocity characteristics of over 6000 structures, finding an average velocity of approximately $1 \mathrm{~km} \mathrm{~s}^{-1}$, with maximum values of $7 \mathrm{~km} \mathrm{~s}^{-1}$. Furthermore, merging magnetic bright points were found to have considerably higher velocities, and significantly longer lifetimes, than isolated structures. By implementing a new and novel technique, we were able to estimate the background magnetic flux of our observational data, which is consistent with a field strength of $400 \mathrm{G}$.
\end{abstract}

Key words: Sun: activity - Sun: evolution - Sun: photosphere

Online-only material: animations, color figure

\section{INTRODUCTION}

Granulation forms the instantly recognizable "patchwork" pattern of the solar photosphere and dominates quiet Sun regions. The dark intergranular lanes are formed as a result of convective downflows and are the regions where magnetic bright points (MBPs) can be observed. It is the horizontal transfer of magnetic flux from the center of granules, and into the lanes, that forms MBPs. In the MBPs, the magnetic flux clumps together to form small magnetic concentrations with field strengths of the order of a kilogauss (Stenflo 1985; Solanki 1993). These are some of the smallest features currently observable on the solar surface, and due to their dynamic nature, can provide a conduit for the transfer of kinetic energy into the upper solar atmosphere (de Wijn et al. 2009).

Due to the increased computational power available in recent years, significant progress has been made in understanding the complex interplay between convective energy transport and radiation in these concentrated magnetic elements. MBPs are best observed as strong intensity enhancements in $G$ band intensity images (Muller \& Roudier 1984; Berger \& Title 1996) which have intensity peaks between 0.8 and 1.8 times the mean photospheric intensity (Sánchez Almeida et al. 2004; Langhans et al. 2002). These enhancements are due to an increase in continuum intensity, caused by the continuum formation layer being depressed into the deeper, hotter layers of the solar photosphere, where regions of strong magnetic field concentrations and reduced $\mathrm{CH}$ abundances exist (Steiner et al. 2001; Shelyag et al. 2004).

The significance of MBPs on solar atmospheric energy transport and dynamics has led to several studies on their area coverage and size distribution. Sánchez Almeida et al. (2004) found that MBPs have a minor axis of $135 \mathrm{~km}$, while Wiehr et al. (2004) determined a predominant diameter of $160 \pm 20 \mathrm{~km}$. In a recent study, Crockett et al. (2010) utilized high spatial resolution observations and magnetohydrodynamic (MHD) simulations to conclude that the MBP area distribution peaks at $\approx 45,000 \mathrm{~km}^{2}$, with a sharp decrease in occurrence for smaller surface areas. The area of the smallest MBPs is defined by the width of the intergranular lanes, which is in turn limited by the balance of radiative and convective energies in the magneto-convective processes.

MBP velocities are induced by the expansion of granules and are typically in the range of $1-3 \mathrm{~km} \mathrm{~s}^{-1}$ (Berger \& Title 1996; Utz et al. 2010). It has been shown by Choudhuri et al. (1993) that magnetic footpoints with velocities greater than $\sim 2 \mathrm{~km} \mathrm{~s}^{-1}$ can excite magnetosonic kink waves, which can transport sufficient energy to heat the localized quiet corona, under a two-layer atmospheric approximation. Also, recent observations (Bonet et al. 2008; Wedemeyer-Böhm \& Rouppe van der Voort 2009) and simulations (Shelyag et al. 2011b) suggest the presence of vortex motions in the photosphere, with a possible connection to the spiral tracks of MBPs and chromospheric swirls. Very recent studies by Shelyag et al. (2011a) and Fedun et al. (2011) have indicated how vortex motions generate a significant amount of Poynting flux directed outward from the photosphere, and, as a result, may be the source of various observed MHD wave modes.

In this Letter, we use high spatial and temporal resolution observations, in addition to numerical simulations, to determine the velocity distribution of a large sample of MBPs. The observations and numerical simulations are described in Section 2, while the methodology used and the values obtained for the velocities of MBP structures are detailed in Section 3. As our tracking algorithm can detect and monitor bright point chains, as well as isolated brightenings and merger events, we believe that this is a unique study of the dynamics of MBPs in the solar photosphere. Differences between the velocity characteristics of non-merging MBPs, and those that undergo mergers with other bright points, are discussed in Section 3. Finally, our concluding remarks are given in Section 4.

\section{OBSERVATIONS AND NUMERICAL SIMULATIONS}

The data employed in this study were obtained using the Rapid Oscillations in the Solar Atmosphere (ROSA; Jess et al. 2010) instrument, which is installed as a common-user facility at the $76 \mathrm{~cm}$ Dunn Solar Telescope, in New Mexico, USA. 


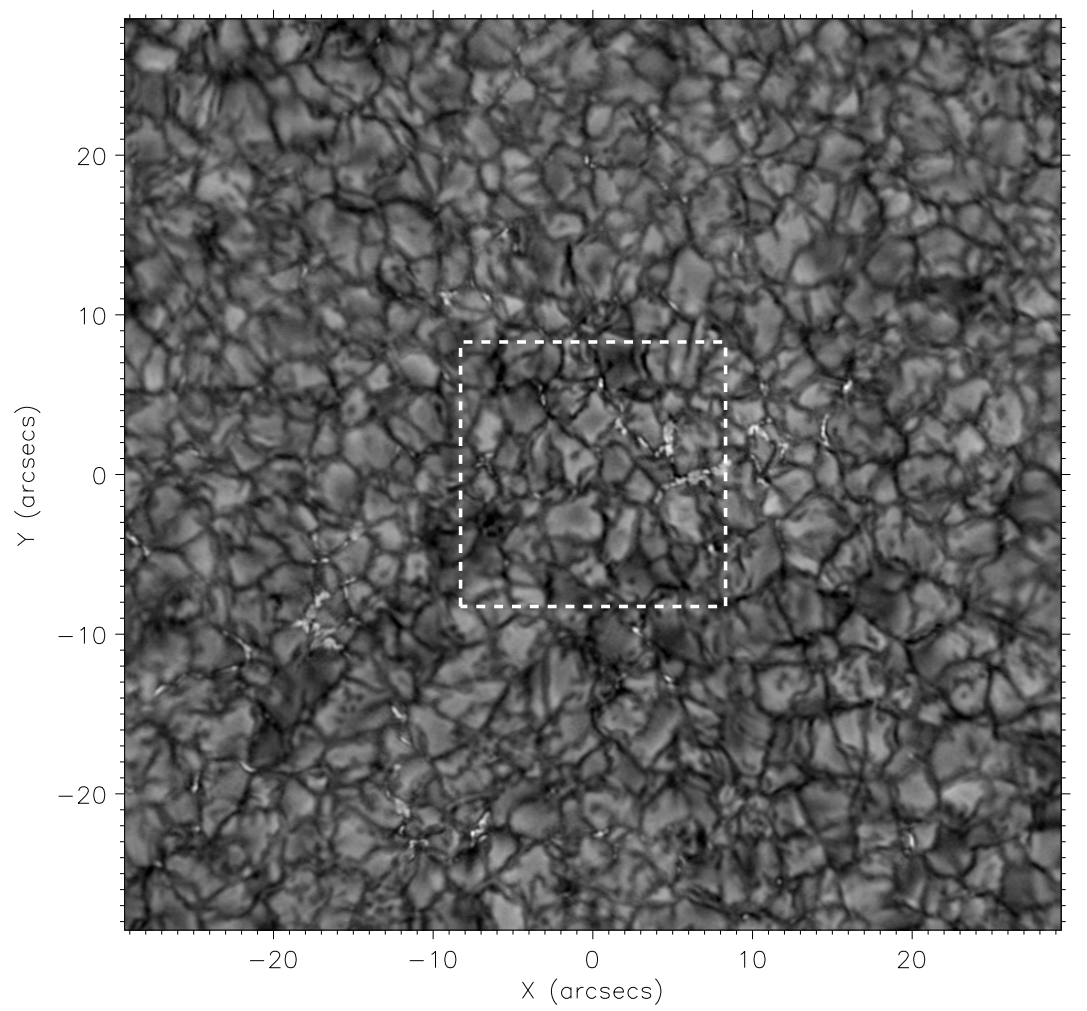

Figure 1. Full $70^{\prime \prime} \times 70^{\prime \prime}$ ROSA field of view of the observational $G$-band data used in this study. The dashed white box indicates a $16^{\prime \prime} \times 16^{\prime \prime}$ region which is expanded in Figure 2(a). The axes are in heliocentric arcseconds, with $(0,0)$ representing the center of the solar disk.

Observations were obtained during a period of excellent seeing on 2009 May 28, using a $9.2 \AA$ wide filter centered at $4305 \AA$ ( $G$ band). We observed a $70^{\prime \prime} \times 70^{\prime \prime}$ quiet Sun region at disk center for $\sim 50$ minutes, achieving diffraction-limited imaging with $0^{\prime}$.069 pixel $^{-1}$. The images were reconstructed using Speckle algorithms (Wöger et al. 2008), while image de-stretching was performed using a $40 \times 40$ grid (equating to $\mathrm{a} \approx 1^{\prime \prime}$.7 separation between spatial samples; Jess et al. 2007, 2008). These processes were implemented to remove the effects of atmospheric seeing from the data set. $G$-band images were taken at a raw cadence of $0.033 \mathrm{~s}$, while after speckle reconstruction the cadence was reduced to $0.528 \mathrm{~s}$. Reconstructed images were then binned into consecutive groups of four to improve the signal to noise and reduce the overall volume of the data set, providing a final image cadence of $2.1 \mathrm{~s}$.

Simulated $G$-band images were produced using the detailed radiative transport technique described by Shelyag et al. (2004), with the solar photospheric magneto-convection models for the radiative transport calculations provided by the MURaM radiative MHD code (Vögler et al. 2005). A computational domain of size $12 \times 12 \times 1.4 \mathrm{Mm}^{3}$ was employed for the simulations and resolved by $480 \times 480 \times 100$ grid cells, providing a horizontal two-pixel resolution of $50 \mathrm{~km}$. The level corresponding to the visible solar surface is located approximately $600 \mathrm{~km}$ below the upper boundary of the domain. Side boundaries of the domain are periodic, while the upper boundary is closed for vertical and stress-free horizontal plasma motions and the bottom boundary is transparent. The numerical solution is stabilized against numerical instabilities using hyperdiffusive source terms (Caunt \& Korpi 2001; Vögler et al. 2005; Shelyag et al. 2008).

In the initial stage of the numerical simulation, we use a well-developed non-magnetic photospheric convection snapshot, where we introduce uniform unipolar vertical magnetic fields of $200 \mathrm{G}$ and $400 \mathrm{G}$, representing two different levels of solar magnetic activity. Following the injection of magnetic fields into the simulation, they quickly become horizontally transferred into the intergranular lanes, creating magnetic field concentrations with a strength of up to $2 \mathrm{kG}$ at the photospheric level. After a few convective turnover timescales, we record 219 and 177 magneto-convection snapshots, at $200 \mathrm{G}$ and $400 \mathrm{G}$, respectively, for further analysis. To obtain the $G$-band intensities emerging from the simulated photospheric snapshots, a spectral band in the wavelength range from $4295 \AA$ to $4315 \AA$, which contains $241 \mathrm{CH}$ molecular lines and 87 atomic lines, was calculated for each vertical ray in the snapshots and convolved with a filter function centered at $4305 \AA$ with a width of $9.2 \AA$.

\section{ANALYSIS AND RESULTS}

The MBPs analyzed in this Letter were detected using the algorithm developed by Crockett et al. (2010). This automated algorithm uses the intergranular lanes to produce a binary map of bright features. A compass search is employed to disentangle features within the lanes, i.e., MBPs, from bright granules. Finally, intensity thresholding across the feature is used to determine more accurately the size and shape of the MBP. Longlived features are then established, and stabilized, by examining subsequent frames to observe the MBP's evolution in time. More details of these procedures may be found in Crockett et al. (2010).

Figure 1 shows the full ROSA field of view, while Figure 2(a) shows an expanded version of the $16^{\prime \prime} \times 16^{\prime \prime}$ region highlighted in Figure 1, together with sample images of the $200 \mathrm{G}$ (Figure 2(b)) and $400 \mathrm{G}$ (Figure 2(c)) simulations. The algorithm produces an outline of the center of gravity of each MBP observed, as well as a direct measurement of the area of each feature. A $30 \mathrm{~s}$ cutoff was employed to remove those MBPs which are short-lived and unlikely to have a significant horizontal velocity component, as 
(a)
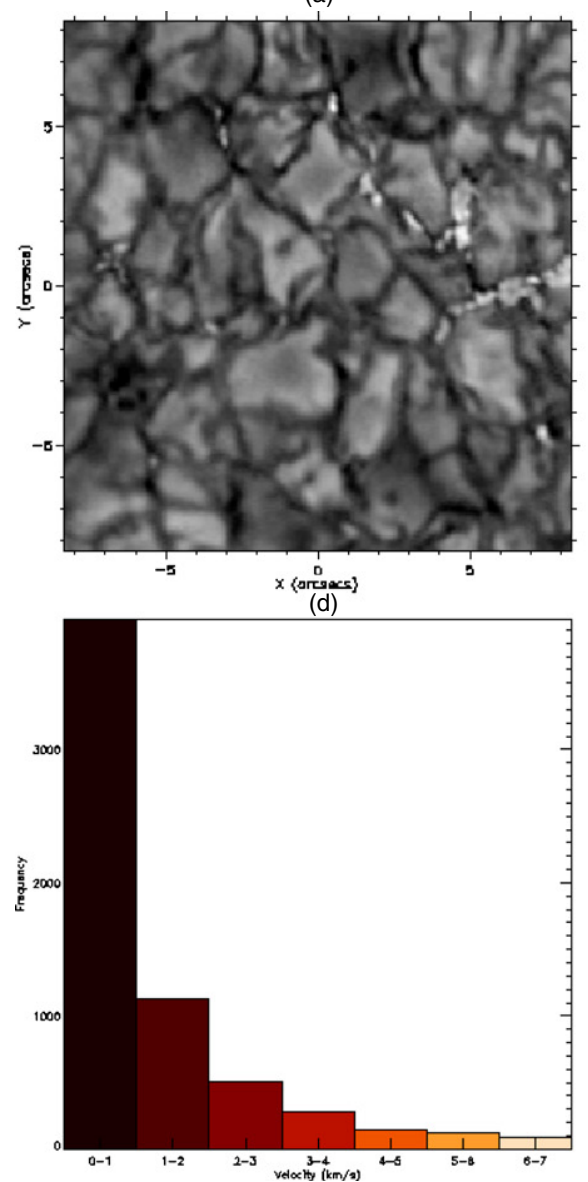

(b)
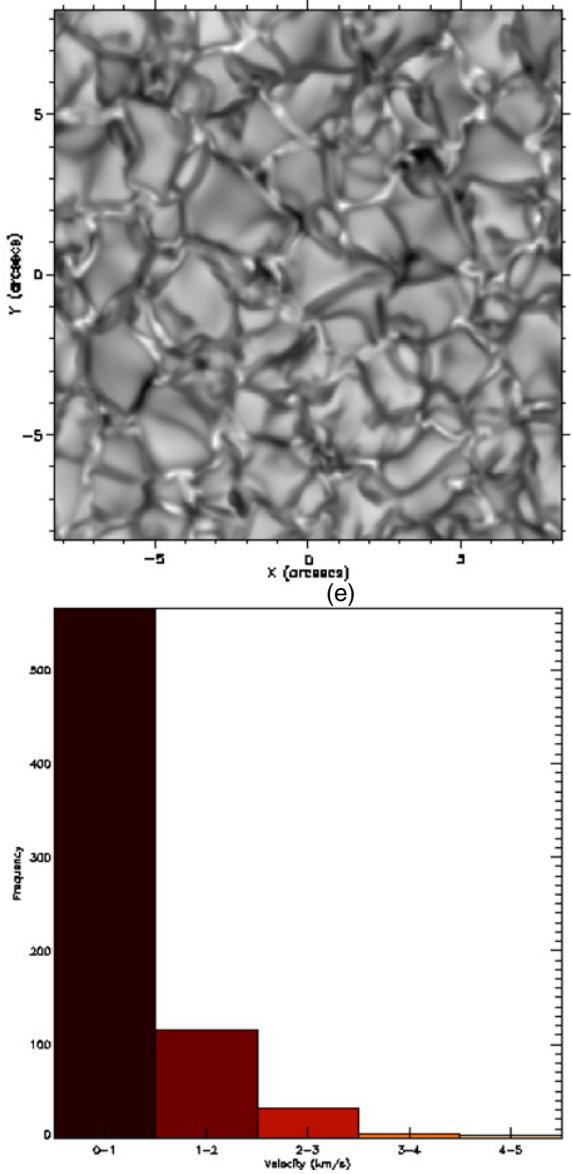

(c)
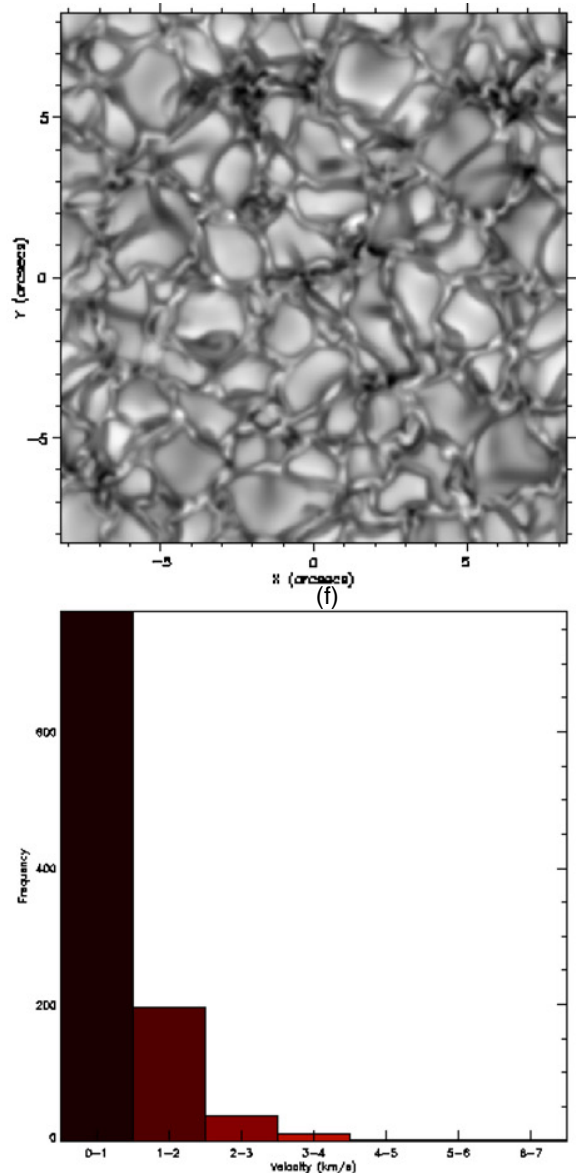

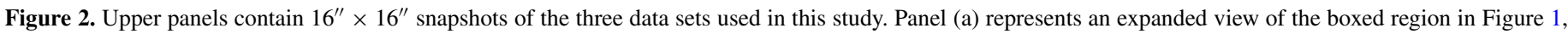

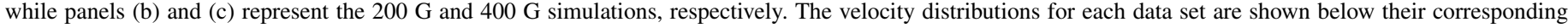
snapshot in panels (d)-(f).

(A color version of this figure is available in the online journal.)

Table 1

Summary of MBP Characteristics

\begin{tabular}{lccc}
\hline \hline Data Set & ROSA Observations & 200 G Sims & $400 \mathrm{G} \mathrm{Sims}$ \\
\hline FOV size & $70^{\prime \prime} \times 70^{\prime \prime}$ & $16^{\prime \prime} \times 16^{\prime \prime}$ & $16^{\prime \prime} \times 16^{\prime \prime}$ \\
Average cadence (s) & 2.112 & 8.7 & 6.7 \\
Number of MBPs detected & 6236 & 721 & 1024 \\
Mean lifetime (s) & 91 & 0.6 & 168 \\
Average velocity $\left(\mathrm{km} \mathrm{s}^{-1}\right)$ & 1.0 & 4.1 & 0.65 \\
Maximum velocity $\left(\mathrm{km} \mathrm{s}^{-1}\right)$ & 7.0 & $4 \%$ & 6.2 \\
Percentage of detections that merge & $21 \%$ & 0.9 & $20 \%$ \\
Average velocity of mergers (km s & \\
Average lifetime of mergers (s) & 1.6 & 227 & 0.8 \\
\end{tabular}

well as assisting the removal of any false detections that may adversely affect the results. See Animation 1 and 2 in the online version of the journal. The first depicts the tracking capabilities of the algorithm for an individual MBP throughout its $336 \mathrm{~s}$ lifetime, while the second displays how an MBP from the $200 \mathrm{G}$ MHD simulation is tracked.

Our observed $G$-band data set included a total of 6236 MBPs with lifetimes exceeding $30 \mathrm{~s}$. These MBPs were found to have an average velocity of approximately $1 \mathrm{~km} \mathrm{~s}^{-1}$ and a mean lifetime of $91 \mathrm{~s}$. The highest velocity recorded in this study was $7 \mathrm{~km} \mathrm{~s}^{-1}$, a value comparable to the photospheric sound speed, while the longest MBP lifetime observed was approximately 20 minutes. Similar values were obtained when the same method- ology was applied to the numerical simulations. A total of 721 MBPs were detected in the $16^{\prime \prime} \times 16^{\prime \prime} 200 \mathrm{G}$ simulation, with an average velocity of $0.6 \mathrm{~km} \mathrm{~s}^{-1}$, a maximum of $4.1 \mathrm{~km} \mathrm{~s}^{-1}$, an average lifetime of $108.2 \mathrm{~s}$, and a maximum of just over 11 minutes. In the $400 \mathrm{G}$ simulation, we detected $1024 \mathrm{MBPs}$, with an average velocity of $0.65 \mathrm{~km} \mathrm{~s}^{-1}$ and a maximum of $6.2 \mathrm{~km} \mathrm{~s}^{-1}$, an average lifetime of $168.4 \mathrm{~s}$ and a maximum of nearly 17 minutes. Histograms indicating the distributions of velocities for all three data sets are shown in Figures 2(d)-(f), with the results summarized in Table 1. A dependence of the velocity distribution on the average magnetic field strength (and thus on the solar magnetic activity level) suggests an alternative, and novel, way of estimating the net magnetic flux in the 
solar photosphere. Hence, as the best agreement with our observations is found for the $400 \mathrm{G}$ simulation, we can infer the average magnetic flux of our observations as being close to 400 G. Small discrepancies between our observed and simulated data sets may be due to the large MBP sample present in our observations (6236 versus 1024).

The distribution of velocities, for both the observed and simulated data sets, is shown as histograms in Figures 2(d)-(f). These demonstrate that the majority of MBPs have velocities between 0 and $1 \mathrm{~km} \mathrm{~s}^{-1}$, while some MBPs (i.e., $30 \%$ in the observations, $6 \%$ in the $200 \mathrm{G}$ simulated data, and $6 \%$ in the $400 \mathrm{G}$ ) have velocities in excess of $2 \mathrm{~km} \mathrm{~s}^{-1}$. The considerable fraction of MBPs with velocities greater than $2 \mathrm{~km} \mathrm{~s}^{-1}$ is important, as rapid bursts in their motion may induce magnetosonic kink waves (Choudhuri et al. 1993). As shown previously, these kink waves may act as a conduit for imparting energy into the upper solar atmosphere.

Although there is considerable agreement between the results obtained in the observed and simulated data, there are some slight discrepancies that need to be addressed for a more complete picture. For example, the maximum velocity, the maximum lifetime, and the proportion of MBPs with velocities greater than $2 \mathrm{~km} \mathrm{~s}^{-1}$ are all smaller in the simulated data sets. A possible reason is that the net (or average) magnetic field applied within the computational domain remains constant throughout the $200 \mathrm{G}$ or $400 \mathrm{G}$ time series, whereas the flux present in the observed data may constantly change with time. This dynamic nature of flux generation and annihilation could lead directly to the higher velocity distributions detected for the observed MBPs.

An alternative algorithm based on local correlation tracking (LCT; Welsch et al. 2004; Matloch et al. 2010) was applied to the observations and simulated time series, and yielded similar velocity characteristics to within $0.3 \mathrm{~km} \mathrm{~s}^{-1}$. A significant number of MBPs, $\approx 21 \%$ of those detected in the observations, were involved with a merging event. Only a few mergers (31) are observed in the $200 \mathrm{G}$ simulation, whereas 207 are detected in the $400 \mathrm{G}$ simulation. Comparisons with an active region area would be a useful study in the future to observe how the frequency of merging events in such a feature compares to the quiet Sun region discussed here.

There is only a slight difference between the velocities obtained for merging events and those of isolated MBPs. The average velocities evaluated for all the merging events in the observations were found to be $1.6 \mathrm{~km} \mathrm{~s}^{-1}$, slightly above the measured value for isolated MBPs. However, the average lifetime of the merging MBPs is $201.2 \mathrm{~s}$, more than twice the value for non-merging MBPs. These results are echoed in the values for the $200 \mathrm{G}$ simulated data set, although only 31 mergers were witnessed out of the 721 detections. Here, the average velocity of the merging MBPs is $0.9 \mathrm{~km} \mathrm{~s}^{-1}$, with an average lifetime of $227 \mathrm{~s}$. For the $400 \mathrm{G}$ simulation time series, the average velocity was found to be $0.8 \mathrm{~km} \mathrm{~s}^{-1}$, with an average lifetime of a merging MBP of 329 s (determined over a total of 207 merging events). These results show that there is little difference in the velocity of a merging MBP when compared with its non-merging counterpart. However, the former do appear to have longer lifetimes. This effect may arise due to an increase in stability of the MBPs when they undergo a merger. One possibility to account for the marginal elevation of velocity characteristics for merging MBPs is the barycenter method used to establish the merging velocity. During a merger, the shift of the center of gravity as the MBPs unite may lead to a larger recorded velocity value. This may also explain why we see such a high maximum velocity in the observed data set. It is most likely an artifact of MBP geometry changes, which induce a considerable motion on the barycenter of the MBP structure. Thus, some velocities may be overestimated by this method. However, employing LCT methods on the same MBP groups indicate that a fraction of these structures do in fact demonstrate velocities comparable to the sound speed.

\section{CONCLUDING REMARKS}

We have used high-resolution observations, and state-of-theart MHD simulations, to determine the velocity distribution of MBPs on the solar surface. The average velocity established from our observations is $1 \mathrm{~km} \mathrm{~s}^{-1}$, averaged over a total of $6236 \mathrm{MBP}$ structures, with a typical lifetime of $91 \mathrm{~s}$. A total of 721 MBPs were tracked in the $200 \mathrm{G}$ numerical simulation, with an average velocity of $0.6 \mathrm{~km} \mathrm{~s}^{-1}$ and a mean lifetime of $108.2 \mathrm{~s}$. The slight differences between the observed and $200 \mathrm{G}$ simulated data sets can be attributed to the initial value of magnetic field applied to the MHD simulation. Using an initial magnetic field of $400 \mathrm{G}$, the subsequent numerical simulation yielded values closer to the observational distribution. The average velocity and average lifetime of the $1024 \mathrm{MBPs}$ detected in the $400 \mathrm{G}$ data are $0.65 \mathrm{~km} \mathrm{~s}^{-1}$ and $168.4 \mathrm{~s}$, respectively. When LCT was applied to each data set, the difference in MBP velocities was found to be $\approx 0.3 \mathrm{~km} \mathrm{~s}^{-1}$, showing a level of consistency between the two independent techniques.

For the merging events witnessed in the observational data set, the average velocity was found to be $1.6 \mathrm{~km} \mathrm{~s}^{-1}$, with a mean lifetime of $201.2 \mathrm{~s}$. The $200 \mathrm{G}$ simulated data set only produced 31 merger events, with an average velocity of $0.9 \mathrm{~km} \mathrm{~s}^{-1}$ and mean lifetime of $227 \mathrm{~s}$. By contrast, the $400 \mathrm{G}$ simulation had 207 mergers, with an average velocity of $0.8 \mathrm{~km} \mathrm{~s}^{-1}$ and mean lifetime of $329 \mathrm{~s}$. All of the data sets show that merging events exhibit longer average lifetimes, which we believe is a result of greater MBP stability as a result of the merger. Although the $200 \mathrm{G}$ simulation produces similar MBP lifetimes, our data are most consistent with the $400 \mathrm{G}$ simulation in both appearance and in MBP merger lifetimes. We intend to expand on this study by observing the velocity characteristics of MBPs and merger events in the vicinity of an active region.

Importantly, we have presented a novel technique for estimating the average photospheric magnetic flux through comparison of observations and MHD simulations. By scaling the initial magnetic flux injected into the numerical simulation to replicate the MBP dynamics established in our observational time series, we can directly estimate the average background magnetic flux in the solar feature studied, without the necessity of vector magnetograms. Following this methodology, we find that our observational data are consistent with an average magnetic flux of $\sim 400 \mathrm{G}$.

This work has been supported by the UK Science and Technology Facilities Council (STFC). Observations were obtained at the National Solar Observatory, operated by the Association of Universities for Research in Astronomy, Inc. (AURA), under cooperative agreement with the National Science Foundation. P.H.K. thanks the Northern Ireland Department for Employment and Learning for a PhD studentship. D.B.J. thanks the STFC for the award of a Post-Doctoral Fellowship. Effort sponsored by the Air Force Office of Scientific Research, Air Force Material Command, USAF under grant number FA8655-09-13085. 


\section{REFERENCES}

Berger, T. E., \& Title, A. M. 1996, ApJ, 463, 365

Bonet, J. A., Márquez, I., Sánchez Almeida, J., Cabello, I., \& Domingo, V. 2008, ApJ, 687, L131

Caunt, S. E., \& Korpi, M. J. 2001, A\&A, 369, 706

Choudhuri, A. R., Dikpati, M., \& Banerjee, D. 1993, ApJ, 413, 811

Crockett, P. J., Mathioudakis, M., Jess, D. B., et al. 2010, ApJ, 722, L188

de Wijn, A. G., Stenflo, J. O., Solanki, S. K., \& Tsuenta, S. 2009, Space Sci Rev., 144, 275

Fedun, V., Shelyag, S., Verth, G., Mathioudakis, M., \& Erdélyi, R. 2011, Ann. Geophys., 29, 1029

Jess, D. B., Andić, A., Mathioudakis, M., Bloomfield, D. S., \& Keenan, F. P. 2007, A\&A, 473, 943

Jess, D. B., Mathioudakis, M., Christian, D. J., et al. 2010, Sol. Phys, 261, 363

Jess, D. B., Mathioudakis, M., Crockett, P. J., \& Keenan, F. P. 2008, ApJ, 688, L119

Langhans, K., Schmidt, W., \& Tritschler, A. 2002, A\&A, 394, 1069

Matloch, Ł., Cameron, R., Shelyag, S., Schmitt, D., \& Schüssler, M. 2010, A\&A, 519, A52
Muller, R., \& Roudier, T. 1984, Sol. Phys., 94, 33

Sánchez Almeida, J., Márquez, I., Bonet, J. A., Domínguez Cerdeña, I., \& Muller, R. 2004, ApJ, 609, L91

Shelyag, S., Fedun, V., \& Erdélyi, R. 2008, A\&A, 486, 655

Shelyag, S., Fedun, V., Keenan, F. P., Erdélyi, R., \& Mathioudakis, M. 2011a, Ann. Geophys., 29, 883

Shelyag, S., Keys, P. H., Mathioudakis, M., \& Keenan, F. P. 2011b, A\&A, 526, A5

Shelyag, S., Schüssler, M., Solanki, S. K., Berdyugina, S. V., \& Vögler, A. 2004, A\&A, 427, 335

Solanki, S. K. 1993, Space Sci. Rev., 63, 1

Steiner, O., Hauschildt, P. H., \& Bruls, J. 2001, A\&A, 372, L13

Stenflo, J. O. 1985, Sol. Phys., 100, 189

Utz, D., Hanslmeier, A., Muller, R., et al. 2010, A\&A, 511, A39

Vögler, A., Shelyag, S., Schüssler, M., et al. 2005, A\&A, 429, 335

Wedemeyer-Böhm, S., \& Rouppe van der Voort, L. 2009, A\&A, 507, L9

Welsch, B. T., Fisher, G. H., Abbett, W. P., \& Regnier, S. 2004, ApJ, 610, 1148

Wiehr, E., Bovelet, B., \& Hirzberger, J. 2004, A\&A, 422, L63

Wöger, F., von der Lühe, O., \& Reardon, K. 2008, A\&A, 488, 375 